Journal Club

Editor's Note: These short, critical reviews of recent papers in the Journal, written exclusively by graduate students or postdoctoral fellows, are intended to summarize the important findings of the paper and provide additional insight and commentary. For more information on the format and purpose of the Journal Club, please see http://www.jneurosci.org/misc/ifa_features.shtml.

\title{
Tau Pathology as a Cause and Consequence of the UPR
}

\author{
Brian J. Stoveken \\ University of Texas Health Science Center at San Antonio, San Antonio, Texas 78229 \\ Review of Abisambra et al.
}

The endoplasmic reticulum (ER) is a site of protein processing and quality control in the cell, replete with mechanisms for recognizing misfolded proteins and resolving proteotoxic stress. These control mechanisms are collectively known as the ER unfolded protein response (UPR; reviewed by Schröder and Kaufman, 2005). UPR signal transduction pathways are orchestrated by the coordinated actions of three key transmembrane proteins: inositol-requiring enzyme $1 \alpha(\operatorname{IRE} 1 \alpha)$, activating transcription factor 6 (ATF6), and protein kinase RNA-like ER kinase (PERK). Together with the ER chaperone $\mathrm{BiP}$, which helps ensure proper protein folding, these factors mediate a rapid and multifaceted response to the accumulation of unfolded proteins in the ER lumen. When activated, the UPR initially induces selective suppression of protein translation, and elevates the expression of chaperones and antioxidant proteins. Additionally, the UPR promotes the stepwise removal of persistently misfolded proteins through ER-associated degradation (ERAD) and the ubiquitin proteasome system (Schröder and Kaufman, 2005). However, chronic activation of the UPR

\footnotetext{
Received July 11, 2013; revised Aug. 7, 2013; accepted Aug. 8, 2013.

I thank Dr. James D. Lechleiter for his encouragement, detailed editorial and figure revisions, as well as Dr. Ramaswamy Sharma, Dr. Naomi Sayre, and Dr. Miranda Orr for their helpful commentary and review of the manuscript. This work was supported by a doctoral fellowship to B.J.S. from the Glenn Foundation for Medical Research.

Correspondence should be addressed to Brian J. Stoveken, University of Texas Health Science Center, South Texas Research Facility, Room 207, 8403 Floyd Curl Drive, San Antonio, TX 78229-3904. E-mail: stoveken@uthscsa.edu.

DOI:10.1523/JNEUROSCI.2961-13.2013

Copyright $\odot 2013$ the authors $\quad 0270-6474 / 13 / 3314285-03 \$ 15.00 / 0$
}

elicits a transition from these generalized prosurvival responses toward one of cell death via apoptosis. Neurological disorders such as Parkinson's disease, Huntington's disease, amyotrophic lateral sclerosis (ALS), and Alzheimer's disease (AD) feature both UPR activation and ERAD dysfunction (Roussel et al., 2013).

In $\mathrm{AD}$ and other neurodegenerative diseases collectively called tauopathies, the microtubule-associated protein tau becomes hyper-phosphorylated and forms insoluble intracellular aggregates associated with disease progression (reviewed by Spillantini and Goedert, 2013). Although a tremendous amount of research has investigated the role of tau in neurodegeneration, the underlying mechanisms of tau toxicity remain elusive.

In their recent article in The Journal of Neuroscience, Abisambra et al. (2013) offer insight into the nature of tau toxicity by revealing that aberrant soluble tau species impair ERAD and activate the UPR. The authors first demonstrate that the appearance of phosphorylated PERK (pPERK), an early marker of incipient ER stress and UPR activation, accompanies the accumulation of soluble tau in rTg4510 mice. These animals conditionally express P301L mutant human $4 \mathrm{R} 0 \mathrm{~N}$ tau, which has an increased tendency to aggregate and is associated with the heritable tauopathy, frontotemporal dementia (Alonso Adel et al., 2004; Santacruz et al., 2005). At 9 months of age, levels of pPERK, the ER chaperone $\mathrm{BiP}$, and ubiquitinylated proteins were higher in transgenic animals than in controls (Abisambra et al., 2013, their Fig. 1). Doxycycline administration suppressed the expression of soluble tau and concomitantly reduced levels of pPERK (Abisambra et al., 2013, their Fig. 5D-H).

Next, Abisambra et al. (2013) induced expression of wild-type human $4 \mathrm{R} 0 \mathrm{~N}$ tau in HEK cells lacking detectable endogenous tau. Levels of pPERK increased in parallel with soluble transgenic tau, peaking $\sim 72 \mathrm{~h}$ after induction (Abisambra et al., 2013, their Fig. 2A-B). Ubiquitinylated proteins rapidly accumulated before PERK phosphorylation, and BiP continued to accumulate beyond $72 \mathrm{~h}$. Cessation of tau overexpression significantly reduced pPERK levels. These data suggest that $4 \mathrm{R} 0 \mathrm{~N}$ tau expression at least transiently activates the UPR in HEK cells.

Elevated levels of ubiquitinylated proteins accompanied tau accumulation in both HEK cells and rTg4510 animals, and this was also observed in the brains of $\mathrm{AD}$ patients (Abisambra et al., 2013, their Figs. $1 A, 2 A, 3$, and 4 ). The majority of this ubiquitinylated protein pool was not attributable to pathologically phosphorylated cytosolic tau. Notably, in rTg4510 mice and $\mathrm{AD}$ brains, 17 and $31 \%$ of respective ubiquitin immunostaining colocalized with the ER membrane protein calnexin, whereas immunostaining of phosphorylated tau did not (Abisambra et al. 2013, their Figs. 3-4). These observations raised the possibility that general proteasomal degradation and ERAD may be impaired in the context of both experimental murine tauopathy and human $\mathrm{AD}$ tau pathology.

Consistent with this possibility, tau was detected ectopically in microsomes isolated from tau-expressing HEK cells. In rTg5410 mice, tau coimmunoprecipitated 
with two cytosolic components of the ERAD and ubiquitin proteasome machinery: VCP/p97 and Hrd1 (Abisambra et al., 2013, their Fig. 6). CD3 $\delta$ (a protein normally degraded via ERAD) accumulated in microsomal fractions isolated from tau-expressing HEK cells and $\mathrm{rTg} 4510$ animals, indicating ERAD dysfunction. Strikingly, $\mathrm{CD} 3 \delta$ accumulation was also identified in tissue from $\mathrm{AD}$ patients (Abisambra et al. 2013, their Fig. 6K-L). These results implicate tau as a cause of ERAD dysfunction, and in light of previously observed UPR activity, suggest protein accumulation in the ER lumen arising from decreased efflux via ERAD.

The authors suggest that impaired ERAD and UPR activation leads to cell death, potentially directly linking tau and neurodegeneration. There are important caveats to this conclusion, however. First, as mentioned above, the UPR is thought to initially serve a prosurvival function, only transitioning to proapoptotic signaling upon chronic or severe ER stress. In this regard, Abisambra et al. (2013) do not show involvement of classically proapoptotic UPR factors such as TRAF2, CHOP, ATF4, or caspases 12, 9, and 3 (Schröder and Kaufman, 2005). Second, their data do not include an assessment of cell viability following UPR activation by tau. This is unfortunate because wild-type and P301L variants of $4 \mathrm{R} 0 \mathrm{~N}$ tau result in distinct neurological pathologies when overexpressed in the brains of mice: P301L $4 \mathrm{R} 0 \mathrm{~N}$ tau induces neuronal degeneration whereas wild-type $4 \mathrm{R} 0 \mathrm{~N}$ tau does not, despite impairing synaptic function (Hoover et al., 2010). It is noteworthy, therefore, that Abisambra and colleagues found that both P301L and wild-type 4R0N tau similarly activate the PERK arm of the UPR. Collectively, this suggests that the P301L mutation is not completely responsible for the tau-induced ER stress observed in vivo, and that UPR activation in these cases might be ancillary to or independent of overt degeneration.

While the impact of tau-mediated UPR activation on cell viability remains unresolved, the results presented by Abisambra et al. (2013) show that ERAD dysfunction can result from the accumulation of soluble $4 \mathrm{R} 0 \mathrm{~N}$ tau, and that similar ERAD impairment occurs in the brains of AD patients. Furthermore, the reversible impact of tau expression on UPR activation as shown by Abisambra et al. (2013) supports the growing view that soluble tau species actively mediate tau toxicity, in addition to (or perhaps despite)

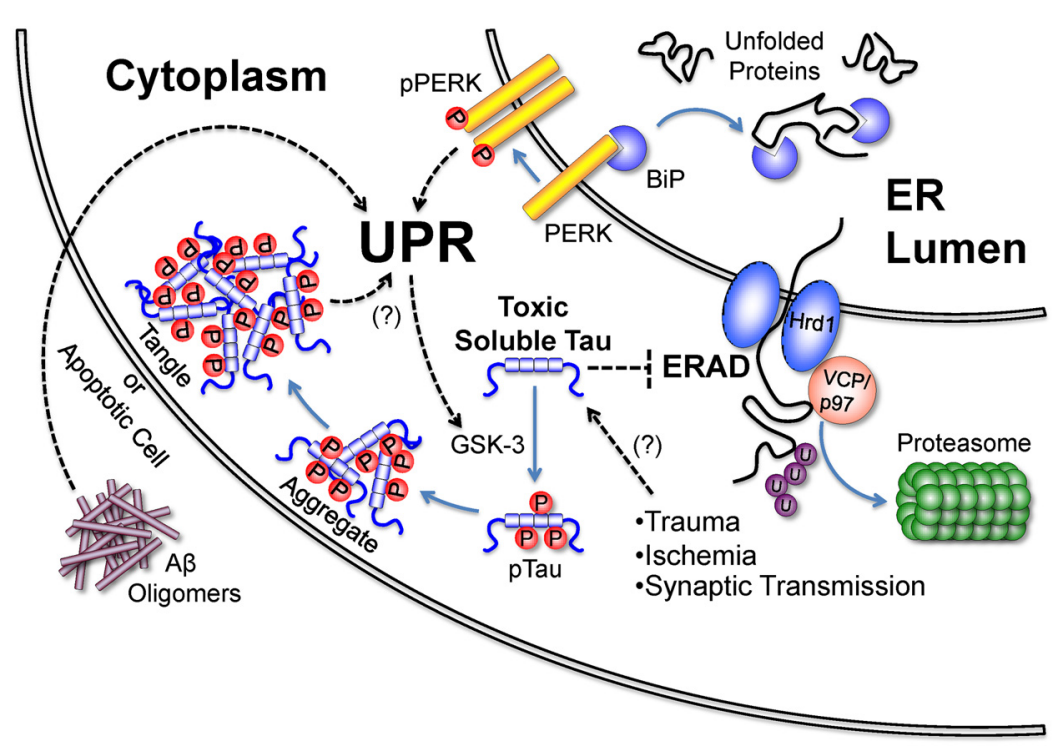

Figure 1. A hypothetical model of tauopathy promoted by ER stress in response to multiple stimuli. Activation of the UPR may result from toxic soluble tau species impairing ERAD through interactions with $\mathrm{Hrd1}$ and VCP/p97, or directly via amyloid- $\beta$ toxicity. Impaired ERAD increases unfolded protein levels in the ER lumen, triggering the UPR by competitively titrating BiP from PERK. Subsequent PERK dimerization and autophosphorylation initiates UPR signaling. The UPR may then direct the misprocessing of tau via selective GSK-3 activation, facilitating tau aggregation. Unresolved UPR activity may alternatively result in cell death via apoptosis. A $\beta$ : Amyloid- $\beta$, UPR: Unfolded Protein Response, GSK-3: Glycogen Synthase kinase 3, p: Phosphate group, U: Ubiquitin.

insoluble tau aggregates (Santacruz et al., 2005).

The results of Abisambra and coworkers differ from those presented by Spatara and Robinson (2010), who showed a lack of UPR induction by wild-type and P301L tau in HEK293 cells. This may reflect several key differences between the two studies. First, Spatara and Robinson evaluated UPR activation 24 and $48 \mathrm{~h}$ after induction of tau expression. Abisambra et al. (2013) show mild, nonsignificant UPR activation at these time points in their cell model (Abisambra et al., 2013, their Fig. $1 A-B)$. Second, Abisambra and colleagues identified UPR induction via post-translational modification of PERK itself. In contrast, Spatara and Robinson (2010) assessed IRE- $1 \alpha$ activity and phosphorylation of the PERK substrate eIF2 $\alpha$. PERK activity can be inferred by the detection of phosphorylated eIF2 $\alpha$ (Schröder and Kaufman, 2005), but Spatara and Robinson show a lack of robust eIF $2 \alpha$ phosphorylation in response to tau expression. It is therefore unfortunate that Abisambra et al. (2013) do not provide evidence of PERK activity in the form of phosphorylated eIF2a at any time point in their study, as their data primarily indicate UPR activation beyond the time points assessed by Spatara and Robinson (2010).

In their discussion, Abisambra and colleagues speculate that tau accumulation seeds the production of additional toxic tau species. Nijholt et al., (2013) provide a potential mechanism by which this might occur. These authors showed that UPR stimulation (indicated by pPERK and downstream eIF2 $\alpha$ phosphorylation) selectively activates glycogen synthase kinase 3 (GSK-3). GSK-3 is a serine/threonine kinase that directly phosphorylates tau at epitopes commonly identified in tau aggregates. As mentioned above, hyperphosphorylated tau is characteristic of tau pathology and hyper-phosphorylation is suggested to promote tau aggregation (Alonso Adel et al., 2004; Jeganathan et al., 2008). The UPR may therefore initiate two key aspects of tau pathology by generating abnormally phosphorylated tau species and promoting tau aggregation. In support of this possibility, Ho et al. (2012) demonstrated tau phosphorylation following UPR activation in primary neuronal cultures from rats.

Notably, the aggregation of tau has been suggested to promote cell survival by sequestering toxic soluble tau species into less toxic insoluble aggregates, similar to mutant huntingtin protein (Arrasate et al., 2004; Santacruz et al., 2005; SpiresJones et al., 2009). One may therefore synthesize the findings of Abisambra et al. (2013) and others as follows: First, tau accumulation activates the UPR by impairing ERAD and activating GSK-3. This leads to persistent hyper-phosphorylation of tau and enhanced aggregation poten- 
tial. If this occurs, then the hallmark tau aggregates seen in $\mathrm{AD}$ and related tauopathies might protect cells by sequestering toxic tau and preventing sustained UPR activation and apoptosis.

A model incorporating the above features is illustrated in Figure 1. This model accommodates multiple initial insults to theCNS, and may help describe the longterm tau-related sequelae observed in chronic traumatic encephalopathy (Hawkins et al., 2013), following transient cerebral ischemia (Wen et al., 2004), or perhaps from amyloid $\beta(\mathrm{A} \beta)$-induced ER stress in $\mathrm{AD}$. Uptake or misprocessing of soluble tau following traumatic brain injury or ischemia could ostensibly lead to tauopathy by causing ER stress. Trans-synaptic propagation of tau pathology, which has been proposed to be mediated by neuronal activity (Pooler et al., 2013) might similarly activate the UPR and promote tau pathology in adjacent cells by transmitting toxic or misprocessed tau. Such stimuli may introduce a cycle of ER stress, altered tau processing, and concomitant aggregation.

The recent association of polymorphisms in the EIF2AK3 locus (encoding PERK) with progressive supranuclear palsy (Hoglinger et al., 2011) has genetically linked the UPR to tauopathy. Abisambra et al. (2013) hypothesize that such genetic variations might alter the expression of PERK, though detailed investigation of haplotype groups carrying the risk alleles has uncovered variations within the coding sequence of PERK, instead suggesting altered function of the protein itself (Stutzbach et al., 2013).

These possibilities require additional investigation. While it is unlikely that UPR activation and ERAD dysfunction are the only factors involved, the publication by Abisambra et al. (2013) highlights the relevance of ER homeostasis and stress response mechanisms in tau pathology. Their work demonstrates that ERAD can be hampered by tau, revealing a straightforward mechanism by which tau impairs cellular function. More broadly, these findings contribute to an increasingly nu- anced view of tau in neurodegenerative disorders; one in which the enigmatic protein might behave as both a potent initiator of ER stress, and perhaps a downstream target of it as well.

\section{References}

Abisambra JF, Jinwal UK, Blair LJ, O’Leary JC 3rd, Li Q, Brady S, Wang L, Guidi CE, Zhang B, Nordhues BA, Cockman M, Suntharalingham A, Li P, Jin Y, Atkins CA, Dickey CA (2013) Tau accumulation activates the unfolded protein response by impairing endoplasmic reticulum-associated degradation. J Neurosci 33:9498-9507. CrossRef Medline

Alonso Adel C, Mederlyova A, Novak M, Grundke-Iqbal I, Iqbal K (2004) Promotion of hyperphosphorylation by frontotemporal dementia tau mutations. J Biol Chem 279: 34873-34881. CrossRef Medline

Arrasate M, Mitra S, Schweitzer ES, Segal MR, Finkbeiner S (2004) Inclusion body formation reduces levels of mutant huntingtin and the risk of neuronal death. Nature 431:805810. CrossRef Medline

Hawkins BE, Krishnamurthy S, Castillo-Carranza DL, Sengupta U, Prough DS, Jackson GR, DeWitt DS, Kayed R (2013) Rapid accumulation of endogenous tau oligomers in a rat model of traumatic brain injury: possible link between traumatic brain injury and sporadic tauopathies. J Biol Chem 288:17042-17050. CrossRef Medline

Ho YS, Yang X, Lau JCF, Hung CHL, Wuwongse S, Zhang Q, Wang J, Baum L, So KF, Chang RCC (2012) Endoplasmic reticulum stress induces tau pathology and forms a vicious cycle: implication in Alzheimer's disease pathogenesis. J Alzheimers Dis 28:839-854. CrossRef Medline

Höglinger GU, Melhem NM, Dickson DW, Sleiman PMA, Wang LS, Klei L, Rademakers $\mathrm{R}$, de Silva R, Litvan I, Riley DE, van Swieten JC, Heutink P, Wszolek ZK, Uitti RJ, Vandrovcova J, Hurtig HI, Gross RG, Maetzler W, Goldwurm S, Tolosa E, et al. (2011) Identification of common variants influencing risk of the tauopathy progressive supranuclear palsy. Nat Genet 43:699-705. CrossRef Medline

Hoover BR, Reed MN, Su J, Penrod RD, Kotilinek LA, Grant MK, Pitstick R, Carlson GA, Lanier LM, Yuan LL, Ashe KH, Liao D (2010) Tau mislocalization to dendritic spines mediates synaptic dysfunction independently of neurodegeneration. Neuron 68:1067-1081. CrossRef Medline

Jeganathan S, Hascher A, Chinnathambi S, Biernat J, Mandelkow EM, Mandelkow E (2008) Proline-directed pseudo-phosphorylation at
AT8 and PHF1 epitopes induces a compaction of the paperclip folding of tau and generates a pathological (MC-1) conformation. J Biol Chem 283:32066-32076. CrossRef Medline

Nijholt DAT, Nölle A, van Haastert ES, Edelijn H, Toonen RF, Hoozemans JJM, Scheper W (2013) Unfolded protein response activates glycogen synthase kinase-3 via selective lysosomal degradation. Neurobiol Aging 34:17591771. CrossRef Medline

Pooler AM, Phillips EC, Lau DHW, Noble W, Hanger DP (2013) Physiological release of endogenous tau is stimulated by neuronal activity. EMBO Rep 14:389-394. CrossRef Medline

Roussel BD, Kruppa AJ, Miranda E, Crowther DC, Lomas DA, Marciniak SJ (2013) Endoplasmic reticulum dysfunction in neurological disease. Lancet Neurol 12:105-118. CrossRef Medline

Santacruz K, Lewis J, Spires T, Paulson J, Kotilinek $\mathrm{L}$, Ingelsson $\mathrm{M}$, Guimaraes $\mathrm{A}$, DeTure $\mathrm{M}$, Ramsden M, McGowan E, Forster C, Yue M, Orne J, Janus C, Mariash A, Kuskowski M, Hyman B, Hutton M, Ashe KH (2005) Tau suppression in a neurodegenerative mouse model improves memory function. Science 309:476-481. CrossRef Medline

Schröder M, Kaufman RJ (2005) The mammalian unfolded protein response. Annu Rev Biochem 74:739-789. CrossRef Medline

Spatara ML, Robinson AS (2010) Transgenic mouse and cell culture models demonstrate a lack of mechanistic connection between endoplasmic reticulum stress and tau dysfunction. J Neurosci Res 88:1951-1961. CrossRef Medline

Spillantini MG, Goedert M (2013) Tau pathology and neurodegeneration. Lancet Neurol 12:609-622. CrossRef Medline

Spires-Jones TL, Stoothoff WH, de Calignon A, Jones PB, Hyman BT (2009) Tau pathophysiology in neurodegeneration: a tangled issue. Trends Neurosci 32:150-159. CrossRef Medline

Stutzbach L, Xie S, Naj A, Albin R, Gilman S, Group PGS, Lee VM, Trojanowski J, Devlin B, Schellenberg G (2013) The unfolded protein response is activated in disease-affected brain regions in progressive supranuclear palsy and Alzheimer's disease. Acta Neuropathol Commun 1:31. CrossRef

Wen Y, Yang S, Liu R, Brun-Zinkernagel AM, Koulen P, Simpkins JW (2004) Transient cerebral ischemia induces aberrant neuronal cell cycle re-entry and Alzheimer's disease-like tauopathy in female rats. J Biol Chem 279: 22684-22692. CrossRef Medline 